\title{
Symbology Development for a 3D Conformal Synthetic Vision Helmet-Mounted Display for Helicopter Operations in Degraded Visual Environment
}

\author{
Patrizia Knabl and Helmut Többen \\ German Aerospace Center (DLR) Institute of Flight Guidance, Lilienthalplatz 7, \\ 38108 Braunschweig, Germany \\ \{Patrizia.Knabl, Helmut. Toebben\} @dlr.de
}

\begin{abstract}
To increase situation awareness for helicopter pilots in poor visibility symbology for a helmet-mounted display was developed. The symbology comprises the conformal presentation of obstacles, route information and threat areas. In an online survey 48 helicopter pilots evaluated the designs from a user-centered perspective and provided comments and suggestions of improvement. The paper presents selected results of the survey and discusses general aspects associated with the use of conformal symbology and helmet-mounted displays.
\end{abstract}

Keywords: helicopter operations, degraded visual environment, synthetic vision systems, helmet-mounted display, conformal symbology.

\section{Introduction}

Degraded visual environment (DVE) is still one of the most challenging and dangerous situations for helicopter pilots. When operating in adverse weather with very poor or no out-the-cockpit visibility spatial disorientation is likely to occur, severely compromising flight safety. Especially in low altitude obstacles like power lines, wind mills and masts quickly become very dangerous to the pilot. This often results in severe accidents, e.g. the recently occurred helicopter collision with a power line in southern Germany [1] and a tower crane in London [2] killing the pilot in both cases.

However, not only poor weather conditions but also surface properties can contribute to a loss of orientation. When operating in desert environment impaired visibility is caused by sand or dust being stirred up by the helicopter's rotor downwash during landing or take-off. This situation is referred to as brownout. Especially in the military field brownout is responsible for multiple incidents and accidents during desert operations. According to the NATO report on Rotary-Wing Brownout Mitigation it is responsible for about $75 \%$ of helicopter mishaps within NATO member countries and the largest cause of rotary-wing airframe loss in the US services alone [3].

Given the high accident rate there is an urgent need to provide the pilot with additional information in DVE to reduce spatial disorientation and workload and increase 
situation awareness. The current paper introduces design solutions on conformal symbology sets for helmet-mounted displays (HMD) and their evaluation from a user-centred perspective. Therefore helicopter pilots assessed the designs in an online-survey.

The aim of the development is to provide an intuitive and well-arranged synthetic presentation of relevant flight information within the pilots' forward field of view that allows for fast and accurate perception of the environment.

\section{Theoretical Background}

Within the national project ALLFlight the DLR Institute of Flight Guidance is working on diminishing the problem of piloting helicopters in DVE conditions [4]. In contrast to currently existing 2D-symbology sets, DLR is developing 3D conformal symbology for a state-of-the-art wide field-of-view binocular HMD. In general a HMD presents information in a monochrome green colour on a semi-transparent visor in front of the pilot's eye. Binocular refers to the presentation of information to both eyes in contrast to monocular displays where information is only presented in front of the dominant eye [5].

The importance of HMD research for rotary-wing aircraft is crucial inasmuch as pilots are usually trained to keep constant visual contact with the outside world even though visibility is poor. Hence staying eyes-out is essential given that pilots frequently operate in very low altitudes or - in the military context - need to perform nap-of-the-earth missions. Thus information should be presented in a way that it does not keep attention on the head-down instruments overly long and does not require frequent attention switching.

However HMD allow for an increased freedom in movements [5], present symbology superimposed and therefore provide the ability to view instrument information and outside scene simultaneously. As a result scanning times as well as eye accommodation when switching between the instrument information and the external scene are reduced [6]. Nevertheless a detriment that is often discussed regarding superimposed symbology is the cost of clutter. It may obscure information in the outside world [7] and impose difficulties in information processing [8]. Combined with a head-tracker the display content can be changed with respect to the line of sight of the pilot. Therefore it allows a conformal presentation of symbology which was found to mitigate the cost of clutter [9]. Conformal -also called scene-linked [10] - refers to "an object that spatially overlies its far domain counterpart" [11]. For example, if an obstacle is presented on the visor it is positioned in the exact line of sight where the actual obstacle in the outside scene is situated. Hence symbols move, appear and disappear on the HMD according to the orientation and movement of both the head and the helicopter. Conformal symbology supports tasks that require divided attention [12] and facilitates the mental integration of the instrument information and the outside scene [13]. In addition conformal symbology was also found to reduce attentional tunneling [9]. 


\section{Display Concepts}

The symbology presented on the HMD is based on information from databases. The concept of ALLFlight foresees the use of sensors as well, but due to the low image quality the sensors are only used to update the database and not to present their imagery directly to the pilot. The symbology designs comprise the conformal depiction of obstacles, route information and threat areas.

\subsection{Obstacle Presentation}

One display objective is the highlighting of obstacles in the outside scene that would normally be difficult to detect. Since the actual appearance of such obstacles is usually very complex it is indispensable to present the synthetic obstacles in a very simplified yet unambiguous way. Obstacles need to be identified easily and fast with little attention required while display clutter has to be kept to a minimum. Each obstacle category is presented as a standardized icon. At first power line masts and wind mills were implemented.

Different designs for power line masts are proposed. Figure 1 depicts a power line mast as an icon with two cross beams (left image) which illustrates the most realistic presentation. Nevertheless it is currently not possible to accurately present the course of the wires that are connected to the masts according to the database information. It is supposed that the horizontal cross beams might lead to confusion by falsely indicating wire direction information that is actually not true. Thus two alternative designs have been developed, a mast with 4 cross beams pointing in each direction (centered image) and one with a circular design (right image).
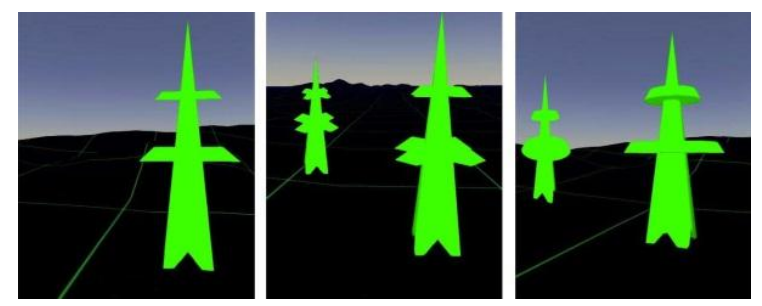

Fig. 1. Power line mast with two cross beams (left), four cross beams (centered) and circular design (right)

In addition three different designs for windmills were developed (Fig. 2). First, the most lifelike presentation depicts a windmill with three rotor blades (left image). Again, since the exact position and orientation of the rotor blades cannot be determined, two alternative designs were implemented. One adds a circle around the blades (centered image) and one adds a globe around the blades (right image). 

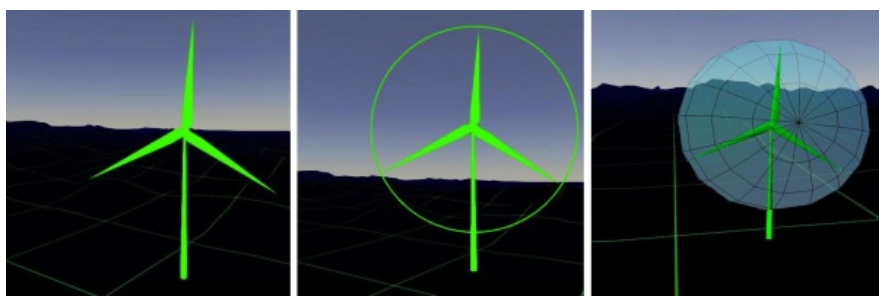

Fig. 2. Windmill with three rotor blades (left), circle (centered) and globe (right)

Moreover it is of interest if pilots need additional information about height and distance relative to the obstacle and if so in what way this information has to be delivered, numerically or graphically. Furthermore information for minimum crossing altitude, vertical range of obstacle depiction and the presentation of visual or auditory alarms are of concern.

\subsection{Route Presentation}

In general route information is intended to assist the pilot in terms of being a navigational aid but not a precise guidance symbology, e.g. a tunnel-in-the-sky [14].

A route is defined by a sequence of pre-determined waypoints. The waypoints are presented as 3-dimensional circles on the ground (Fig. 3). To make them more apparent they also feature a vertical light beam. This is especially important if the next waypoint is hidden due to a terrain elevation. In addition numerous route points are presented between the waypoints. Route points serve as directional cues to the next waypoint, they are depicted as a 3-dimensional triangle placed on the terrain, are equally spaced, follow the elevation and their size is perspective for better distance estimation. Furthermore the top always points in the direction of the next route- and waypoint.

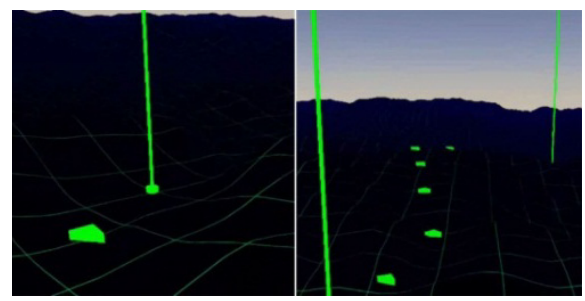

Fig. 3. Waypoint and route point presentation

\subsection{Threat Area Presentation}

The threat area symbology is designed to depict prohibited airspace that must not be entered. Three different threat zone presentations were developed (Fig. 4). First, a threat zone is presented by equidistant crosses on the terrain (left image). The crosses are perspective and follow the terrain elevation. In addition the second threat area 
depiction contains vertical lines arising from these crosses (centered image). When flying alongside the threat zone only the crosses are presented, however if the heading of the helicopter is directed towards the threat zone vertical lines start to arise from the ground and grow taller with decreased distance to the threat zone. Thus the lines rise and fall relative to the flight direction of the helicopter and the resulting severity of the threat. Alternatively a threat zone can be presented as a surface shell, covering the restricted area under a cupola (right image).

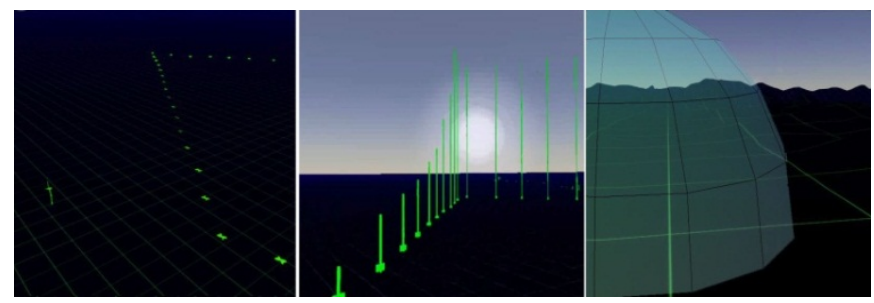

Fig. 4. Threat zone with terrain crosses (left), vertical lines (centered) and surface shell (right)

\section{$4 \quad$ Method}

An online survey was developed using the open source application LimeSurvey Version 1.91. The survey addressed the target group of helicopter pilots. The link to the survey was distributed to several governmental facilities and private helicopter companies.

First pilots were given a short briefing summarizing the aim and content of the survey. In addition they were informed that their statements are handled confidentially. The survey was anonymous, names or email-addresses did not have to be provided. The questionnaire then contained a section on demographic information as well as sections on the presentation of obstacle information, route information and threat area information. The demographic data section for example contained questions about age, sex, nationality, flying experience and prior experience with HMD and conformal symbology. Within the following sections pilots were shown pictures and simulated in-flight videos of the symbology sets. They were then asked to rate the designs with regard to different aspects and provide open comments or suggestions of improvement. After completing the survey they were thanked for their participation and contact information in case of questions and interest in the results was provided. It took approximately 30 minutes to complete the survey. All questions except for open comments were obligatory to proceed in the survey. Only complete data sets were evaluated, hence every question was answered by each pilot.

The data output was exported into SPSS 20.0. Variables were re-coded in a way that high scores denote a high level of agreement. With regard to the on-going discussion about whether rating scales have to be considered as an ordinal or interval scale of measurement it was decided to pursue the more pragmatic approach and follow the rather common practice by interpreting the rating scales on an interval level of measurement [15]. 


\section{$5 \quad$ Results}

\subsection{Participants}

48 male pilots participated in the survey. The age of participants ranged from 23 to 62 years $(\mathrm{M}=43.9, \mathrm{SD}=8.2)$. Nationalities included pilots from Germany, Switzerland, Austria, Israel, USA, Canada, UK and South Africa. 38 were civil, 10 were military pilots. They averaged $1966.4(\mathrm{SD}=1104.1)$ flight hours on their helicopter most frequently operated and $4769.6(\mathrm{SD}=3273.5)$ flight hours in total. 24 pilots were IFRrated. 21 pilots reported having experienced severe spatial disorientation in flight due to DVE once and 10 of them even repeatedly. Furthermore 11 pilots reported having encountered a brownout or whiteout situation in flight once and 23 repeatedly. 13 participants had prior experience with HMD either in real flight or in the simulator averaging 209.3 ( $\mathrm{SD}=315.5$ ) flight hours. In addition 38 pilots had on average 299.2 $(\mathrm{SD}=352.3)$ flight hours experience with night vision goggles. Finally 13 participants reported to have already flown with 3D-conformal symbology averaging 221.3 $(\mathrm{SD}=416.0)$ flight hours.

\subsection{Obstacle Presentation}

Repeated Measures ANOVAs and Bonferroni post-hoc tests were conducted to assess differences in ratings between the designs each on a scale from one (strongly disagree) to four (strongly agree).

\section{Power Line Masts}

In general there is an overall advantage stated in favor for the mast with two crossbeams. 38 (79.2\%) pilots ranked the mast icon with two cross beams as their first preference while six pilots $(12.5 \%)$ favored the circular design and four pilots $(8.3 \%)$ the icon with four cross beams. As their second choice, 25 pilots $(52.1 \%)$ preferred the four-cross-beam icon, $18(37.5 \%)$ the circular design and $5(10.4 \%)$ the mast with two cross beams. In line with the results of the ranking pilots state that they would like to use the design with two beams preferably $(\mathrm{M}=3.2, \mathrm{SD}=0.7)$ rather than the mast with four beams $(\mathrm{M}=2.2, \mathrm{SD}=0.8)$ or the circular mast $(\mathrm{M}=2.2, \mathrm{SD}=0.9)$, $\mathrm{F}(2,94)=22.2, \mathrm{p}=.00$. Besides participants rated the two-cross-beam design $(\mathrm{M}=3.6$, $\mathrm{SD}=0.5)$ to be easier to identify than the four-cross-beam $(\mathrm{M}=2.9, \mathrm{SD}=0.7)$ and the circular $(\mathrm{M}=2.8, \mathrm{SD}=0.9)$ design, $\mathrm{F}(2,94)=23.2, \mathrm{p}=.00$. Furthermore it is rated to be more intuitively understandable $(\mathrm{M}=3.4, \mathrm{SD}=0.6)$ and less complex $(\mathrm{M}=1.7, \mathrm{SD}=0.6)$ compared to the four-cross-beam $(\mathrm{M}=2.7, \mathrm{SD}=0.7 ; \mathrm{M}=2.5, \mathrm{SD}=0.8)$ and the circular $(\mathrm{M}=2.6, \quad \mathrm{SD}=0.8 ; \mathrm{M}=2.4, \quad \mathrm{SD}=0.8)$ mast presentation, $\mathrm{F}(2,94)=20,8, \mathrm{p}=.00$; $\mathrm{F}(2,94)=23.5, \mathrm{p}=.00$. In addition pilots also indicated that the mast with two cross beams produced the least amount of clutter $(\mathrm{M}=2.0, \mathrm{SD}=0.6)$ compared to the fourcross-beam $(\mathrm{M}=2.6, \mathrm{SD}=0.8)$ and circular design $(\mathrm{M}=2.4, \mathrm{SD}=0.8), \mathrm{F}(2,94)=10.2$, $\mathrm{p}=.00$. 


\section{Windmills}

The three-rotor-blade windmill was ranked first by 29 pilots (60.4\%) while 16 (33.3\%) preferred the windmill with the circle and three pilots (6.3\%) favored the windmill with the globe around the blades. Secondly 30 pilots (62.5\%) opted for the windmill with the circle, $15(31.3 \%)$ preferred the regular windmill and three (6.3\%) the windmill with the globe. Results on windmill ratings revealed a clear overall advantage for the de-cluttered conventional windmill with the three rotor blades. Hence pilots would like to use the conventional windmill design $(\mathrm{M}=3.2, \mathrm{SD}=0.7)$ preferably over the windmill with the circle $(\mathrm{M}=2.8, \mathrm{SD}=0.9)$ and the windmill with the globe $(\mathrm{M}=1.8, \mathrm{SD}=0.7), \mathrm{F}(1.7,83.2)=44.9 \mathrm{p}=.00$. Participants indicated that the conventional windmill icon $(\mathrm{M}=3.5, \mathrm{SD}=0.7)$ was significantly easier to identify than the icon with the circle $(\mathrm{M}=3.3, \mathrm{SD}=0.7)$. However there was also a significant advantage in favor for the circle-design compared to the globe $(\mathrm{M}=2.6, \mathrm{SD}=0.8), \mathrm{F}(2,94)=31.2$; $\mathrm{p}=.00$. In addition the de-cluttered windmill was rated to be less complex $(\mathrm{M}=1.7$, $\mathrm{SD}=0.6)$ and more intuitively understandable $(\mathrm{M}=3.5, \mathrm{SD}=0.7)$ compared to the circle design $(\mathrm{M}=2.4, \mathrm{SD}=0.8 ; \mathrm{M}=3.3, \mathrm{SD}=0.6)$ and the globe. Here again the circle design is favored over the globe ( $\mathrm{M}=3.3, \mathrm{SD}=0.8 ; \mathrm{M}=2.5, \mathrm{SD}=0.7), \mathrm{F}(2,94)=81.1, \mathrm{p}=.00$; $\mathrm{F}(2,94)=44.3$. Ratings also indicated the lowest level of clutter for the conventional windmill $(\mathrm{M}=1.8, \mathrm{SD}=0.6)$ compared to the circle $(\mathrm{M}=2.3, \mathrm{SD}=0.7)$ and the globe $(\mathrm{M}=3.1, \mathrm{SD}=0.8), \mathrm{F}(1.7,81.0)=56.8 \mathrm{p}=.00$.

\section{Presentation of Obstacle-Related Information}

Results on the presentation of altitude information revealed that according to 27 pilots (56.3\%) altitude should be indicated by a graphical presentation. 17 pilots (35.4\%) favored a numerical presentation and only four pilots (8.3\%) do not need additional altitude information at all. With regard to the graphical presentation results indicated an overall advantage for presenting an additional altitude marker symbol stated by 42 pilots (87.5\%) compared to a brightness coding of the obstacle which was preferred by only 6 pilots $(12.5 \%)$.

In contrast distance should preferably be indicated by numerical information next to the obstacle stated by 30 pilots $(62.5 \%)$. Only eleven participants (22.9\%) favored a graphical representation while seven $(14.6 \%)$ do not need to see distance information at all. The numerical information should most preferably be presented below the obstacle $(31.3 \%)$ or on the upper left side of the obstacle $(31.3 \%)$.

19 pilots (39.6\%) reported that they need information on minimum crossing altitude. The majority of 27 participants $(56.3 \%)$ stated that obstacles should always be presented independent from vertical range (altitude).

With regard to the presentation of an alarm if the distance to an obstacle is below a certain limit, $26(54.2 \%)$ pilots stated that both a visual and an auditory alarm should be given. 16 (33.3\%) participants favored solely a visual alarm, five $(10.4 \%)$ an auditory alarm and only one pilot $(2.1 \%)$ stated that no alarm should be given. 


\subsection{Route Presentation}

\section{Waypoints}

In general waypoints are rated rather well since 33 pilots $(68.8 \%)$ indicated that they would like to use the design. Furthermore waypoints were rated to increase situation awareness $(85.5 \%)$, be easy to identify $(83.3 \%)$, intuitively understandable $(77.1 \%)$ and not produce too much clutter (79.2\%). In addition 40 pilots (83.3\%) reported that the light beam is useful. Only five pilots (10.4\%) do not want to see waypoints at all. Nevertheless at least $22(45.9)$ pilots agreed to some extent that the presentation is lacking information while on the other hand five pilots (10.4\%) do not want to see waypoints at all.

\section{Route Points}

The majority of $40(83.3 \%)$ pilots agreed that route points would increase situation awareness and are useful $(37,77.1 \%)$. With regard to the design $41(85.5 \%)$ rated them to be easy to identify and $36(75.0 \%)$ to be intuitively understandable. 15 (31.3\%) stated that route points produce too much clutter and 9 (18.8\%) do not want to see them at all. In the end $32(66.7 \%)$ wanted to use the design

\subsection{Threat Zone Presentation}

$20(41.7 \%)$ pilots ranked the surface shell as their first choice, however $16(33.3 \%)$ pilots preferred the representation with the vertical lines and $12(25 \%)$ pilots the representation with the crosses alone. Secondly 19 (39.6\%) pilots favored vertical lines, $15(31.3 \%)$ pilots the crosses and $14(29.2 \%)$ the surface shell.

Participants rated the surface shell to be more intuitively understandable $(M=3.2$, $\mathrm{SD}=2.6)$ than the crosses $(\mathrm{M}=2.8, \mathrm{SD}=0.8), \mathrm{F}(2,94)=6.9, \mathrm{p}=.02$. However the surface shell was also rated highest for producing too much clutter $(\mathrm{M}=2.7, \mathrm{SD}=0.7)$ compared to the cross-design $(\mathrm{M}=2.4, \mathrm{SD}=0.6), \mathrm{F}(2,94)=4.5, \mathrm{p}=.01 .32$ pilots $(66.7 \%)$ stated that the surface shell might capture too much attention while 17 (35.4\%) do not want to see it at all. In contrast 16 pilots (33.4\%) agreed that the crosses alone are not noticeable enough. However also 33 participants (68.7\%) suspected that the vertical lines might capture too much attention and 19 (39.6\%) do not need to see the vertical lines at all. Finally 27 (56.3\%) helicopter pilots would like to use the surface shell design, 26 (54.1\%) the design with the crosses and 23 (47.9\%) the design with the vertical lines.

\section{Discussion}

In summary the survey dealt with the evaluation of symbology designs that have been developed to increase helicopter pilot situation awareness in poor visibility. The demographic statements endorse the relevance of such a development as the majority of pilots reported about having experienced severe spatial disorientation in flight due to DVE or due to brownout and whiteout. These findings are in accordance with the high incident rates stated in the NATO report [3]. 
Altogether the survey revealed some very interesting and useful results. First it becomes clear that there is a common interest in the use of such technology not only in the military field but also for civil applications.

This legitimates further research not only to be looking into expensive high-end applications that are primarily designed for the military context but also into the development of feasible low-cost alternatives for civil implementation.

With regard to the design of icons and symbols it becomes apparent that clutter is still the most crucial concern for pilots. This is reflected especially in the results for obstacle ratings where the de-cluttered designs - the mast with two cross beams and the conventional windmill - were rated best. Furthermore the cost of clutter and symbology overload was also the highest concern reflected in the comments. This confirms the fact that staying eyes-out and being able to see the outside scene is essential for helicopter pilots in any circumstances. On the other hand results were less clear for the threat zone designs. In fact the de-cluttered cross-design scored insignificantly lowest. The result can probably be explained by the fact that the cross-design is also the less salient one.

Nevertheless the need for information about altitude and distance as well as the presentation of an alarm becomes very clear in the results. Here a slightly preference to present altitude in a graphical and distance in a numerical way is shown.

In addition it should be briefly discussed that pilot comments repeatedly concerned the change of colour especially for alerts and threat indications. Nevertheless, as mentioned in chapter 2, symbology can only be depicted in monochrome green. Therefore only the coding of brightness is currently an option, otherwise different alternatives such as blinking or highlighting with a frame have to be tested. However the requests for changing color will be taken into consideration for a possible implementation on a head-down display.

Moreover it became clear in the comments that the presentation of power line wires is crucial. However as mentioned in chapter 3.1 wire directions cannot be precisely depicted from database information. Thus as a next step DLR is also investigating the use of helicopter-mounted sensors to provide real-time information updates in the database, for instance by using a modified laser radar (Hellas) developed by Cassidian.

Finally the outcomes of the survey will now be integrated into the symbology design, implemented into the HMD and furthermore tested in part-task simulations and flight trials.

Acknowledgements. The authors would like to thank all colleagues who participated in the workshops and assisted in the development of the questionnaire for their valuable contributions.

\section{References}

1. http://www.welt.de (January 10, 2013) Retrieved from Hubschrauber stürzt auf Autobahn - Pilot stirbt, http://www.welt.de/vermischtes/ weltgeschehen/article112683378/Hubschrauber-stuerzt-aufAutobahn-Pilot-stirbt.html 
2. AAIB, AAIB Bulletin S1/2013 SPECIAL. Air Accidents Investigation Branch (AAIB), Aldershot, UK (2013)

3. NATO Task Group HFM-162, Rotary-Wing Brownout Mitigation: Technologies and Training. RTO/NATO (2012)

4. Lüken, T., Doehler, H.-U., Lantzsch, R.: ALLFlight - Fusing sensor information to increase helicopter pilot's situation awareness. In: Proceedings of the 36th European Rotorcraft Forum (2010)

5. Velger, M.: Helmet-Mounted Displays and Sights. Artech House, Boston (1998)

6. Yeh, M., Wickens, C., Seagull, F.: Effects of frame of reference and viewing condition on attentional issues with helmet mounted displays. U.S. Army Research Laboratory, Maryland (1998)

7. Yeh, M., Wickens, C., Seagull, F.: Target Cuing in Visual Search: The Effects of Conformality and Display Location on the Allocation of Visual Attention. In: Society, H.F. (ed.) Human Factors, vol. 41(4), pp. 524-542 (1999)

8. Wickens, C.: Attentional Issues in Head-up Displays. In: Harris, D. (ed.) Engineering Psychology and Cognitive Ergonomics, vol. 1, pp. 3-21. Ashgate (1997)

9. McCann, R., Foyle, D.: Scene-linked Symbology to Improve Situation Awareness. In: AGARD Conference Proceedings No. 555. Aerospace Medical Panel Conference on Situation Awareness, pp. 16:1-16:11 (1995)

10. Foyle, D.C., Ahumada, A.J., Larimer, J., Townsend Sweet, B.: Enhanced/Synthetic Vision Systems: Human Factors Research and Implications for Future Systems. SAE Transactions: Journal of Aerospace 101, 1734-1741 (1992)

11. Wickens, C.D., Long, J.: Object versus space-based models of visual attention: Implications for the design of head-up displays. Journal of Experimental Psychology: Applied 1(3), 179-193 (1995)

12. Martin-Emerson, R., Wickens, C.: Superimposition, Symbology, Visual Attention, and the Head-Up Display. Superimposition, Symbology, Visual Attention, and the Head-Up Display 39(4), 581-601 (1997)

13. Wickens, C.: Aviation Displays. In: Tsang, P., Vidulich, M. (eds.) Principles and Practice of Aviation Psychology, pp. 147-200. Lawrence Erlbaum Associates, Mahwah (2003)

14. Alexander, A.L., Wickens, C.D., Hardy, T.J.: Synthetic Vision Systems: The Effects of Guidance Symbology, Display Size, and Field of View. In: Society, H.F. (ed.) Human Factors, vol. 47(4), pp. 693-707 (2005)

15. Bortz, J.: Statistik für Human- und Sozialwissenschaftler, 6th edn. Springer Medizin Verlag, Heidelberg (2005) 